\title{
ZWEIHUNDERTUNDVIERZIG JAHRE NACH SOLON
}

\author{
F. X. Ryan
}

\begin{abstract}
A passage of Demosthenes clearly implies a much higher date for the death of Solon than those recorded by Plutarch; since the tendency of the passage is reinforced by this higher date, one may readily believe that the figure transmitted to us is the one intended by the orator.
\end{abstract}

Es trenne Solon von der Gegenwart ein Zeitraum von etwa 240 Jahren (Dem. 19.251). Diese Aussage begegnet uns in einer Rede, die erst im Jahre 343/2 v. Chr. (Dion. Hal. Amm. 10) gehalten wurde, also gut 250 Jahre nach der Gesetzgebung Solons, auf die sie nach der einhelligen Auffassung der neueren Forschung zu beziehen sei. ${ }^{1}$ Bei dieser Übereinstimmung überrascht es, daß man die Stelle des Demosthenes da, wo man die Gesetzgebung datiert, ${ }^{2}$ ja sogar da, wo man sie herabdatiert, ${ }^{3}$ mit keinem Wort erwähnt. Daß die Zahl trotz der Übereinstimmung noch nicht korrigiert worden ist, liegt daran, daß man dem Redner so einen Rechenfehler zutraut oder ihn unwissend sein läßt. ${ }^{4}$ Jacobys Auslegung des Textes unterscheidet sich insofern von der heutigen nicht, als auch sie Demosthenes gering schätzt. ${ }^{5}$ Ihm zufolge gehe es um die Eroberung von Salamis, die ja vor der Gesetzgebung erfolgt sein soll, so daß Demosthenes hiernach mit der Chronologie erst recht nicht klarkommt. Nach Jacoby weise der Zusammenhang sogar „ganz klar“ auf die Eroberung von Salamis hin. ${ }^{6}$ Freilich werden im folgenden Kapitel (Dem. 19.252) der Abfall der Insel und die dem Solon zugeschriebene Elegie erwähnt, und auch in dem uns interessierenden Kapitel ist von Salamis die Rede. Wenn man aber Jacobys Vorgehensweise nachmacht und die Aussage in ihrem ursprünglichen Zusammenhang sieht, wird man zu einem anderen Schluß kommen müssen als er: Die Stelle des Demosthenes handelt nicht von der Zurückeroberung von Salamis, sondern von der Insel lediglich als dem Aufstellungsort einer Statue Solons.

\footnotetext{
${ }^{1}$ T. J. Cadoux, The Athenian Archons from Kreon to Hypsichides, JHS 68, 1948, 93: „Of these (sc. references), one may be briefly dismissed. Demosthenes, in a speech delivered in 343, stated that it was then 240 years 'since Solon'“; R. Develin, Athenian Officials 684-321 B.C., Cambridge 1989, 37: „Demosthenes 19.251 in 343 speaks of 240 years since Solon“; T. Paulsen, Die Parapresbeia-Reden des Demosthenes und des Aischines. Kommentar und Interpretationen zu Demosthenes, or. XIX, und Aischines, or. II, Trier 1999, 246: „Nimmt man Solons Reformen von 594 als Stichdatum, sind es genau 251 Jahre“; D. M. MacDowell, Demosthenes On the False Embassy (Oration 19), Oxford 2000, 309: „Solon was arkhon in 594/3, which was 251 years before the date of this speech."

${ }^{2}$ R. W. Wallace, The Date of Solon's Reforms, AJAH 8, 1983 [1986], 81-95.

${ }^{3}$ R. Sealey, Zum Datum der solonischen Gesetzgebung, Historia 28, 1979, 238-41.

${ }^{4}$ Cadoux, Archons 93: „This statement, imprecise and unsupported by other evidence as it is, may either have been an unchecked commonplace which Demosthenes did not realise was ten years old, or it may have been due to inaccurate counting-up from the archon-list in the course of hasty preparation for the law-courts"; MacDowell (A. 1) 309: „Possibly the numeral here is corrupt, but more probably $\mathrm{D}$ (emosthenes) did not know the exact date and was just making an estimate."“

${ }^{5}$ F. Jacoby, Apollodors Chronik, Berlin 1902, 172: „selbst wenn Demosthenes hier vom archontate Solons spräche, würde niemand, der die zeugnisse wägt, die oberflächliche angabe eines redners der chronologisch sorgfältigen darstellung des — übrigens doch gleichzeitigen — Aristoteles vorziehen.“

${ }^{6}$ Jacoby, Chronik 172-73 m. A. 18.
} 
Daß das hier in Rede stehende Ehrenbildnis aus Bronze sei, weiß nur eine späte Quelle ([Dion Chrys.] 37.7) zu berichten, die fälschlicherweise von der Aufstellung desselben zu Solons Lebzeiten ausgeht, ${ }^{7}$ da dieser Gewährsmann meint, Solon habe viel auf diese Statue wie auf Statuen im allgemeinen gegeben, aber keine zweite Statue erwähnt, wird er nichts von einer zweiten Statue gewußt haben. Die Statue Solons, die wahrscheinlich erst im vierten Jahrhundert in Athen auf der Agora errichtet wurde, war in der Tat eine Bronzestatue ([Dem.] 26.23, Paus. 1.16.1, Ael. VH 8.16); ${ }^{8}$ der Umstand, daß die Augenzeugenberichte den Ge-

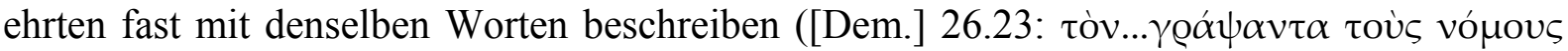

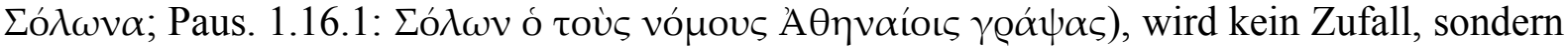
auf die Basisinschrift zurückzuführen sein. Die erklärende Inschrift, mit welcher unsere Statue

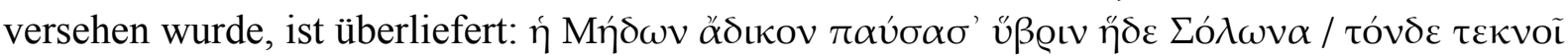

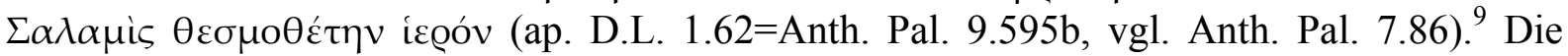
Basisinschrift ist also nicht vor dem Jahr 480 entstanden.

Erwähnt wird unsere Statue zum ersten Mal bei Aischines, der an ihrer Pose, genauer an dem Verbergen der Hand, die würdevolle Redehaltung Solons zu erkennen glaubte: ع $\tilde{u} \gamma \dot{\alpha} \varrho$ oĩ

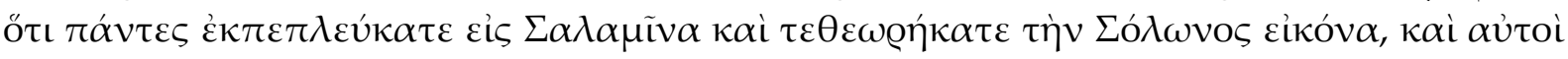

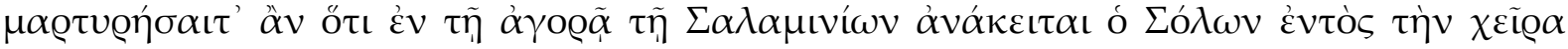

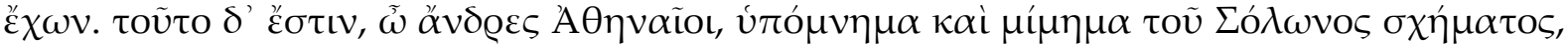

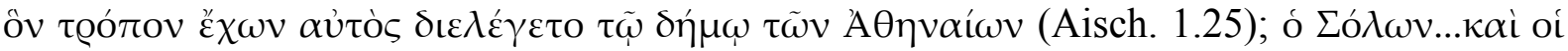

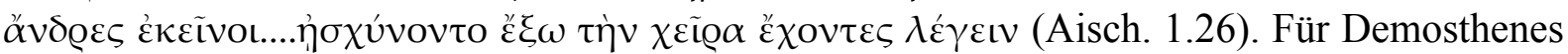
war die von Aischines erteilte Nachhilfe ein gefundenes Fressen, denn die Aufstellung der

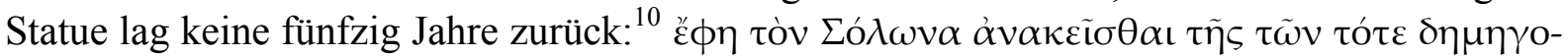

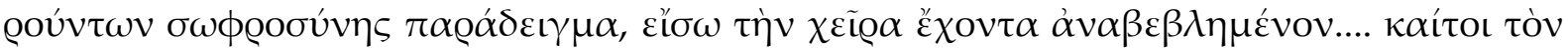

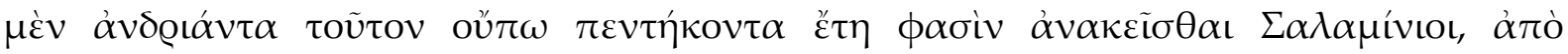

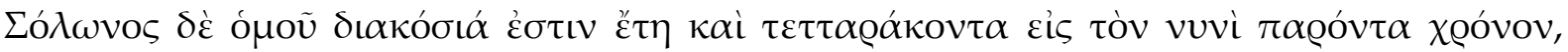

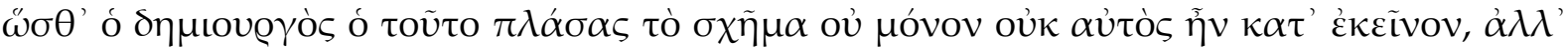

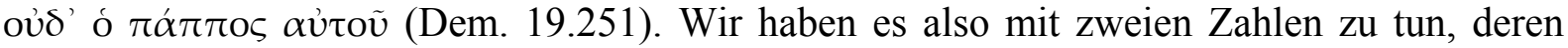
zweite über die erste zu enträtseln ist: Das Intervall von der Aufstellung der Statue auf das aktuelle Jahr betrage knapp 50 Jahre, während das Intervall von Solon um die 240 Jahre ausmache. Die Differenz ist sage und schreibe 190 Jahre. Daraus geht klar hervor, daß der Bildhauer, der Solon die würdevolle Pose einnehmen ließ, keine Rede desselben miterlebt hatte. Der Großvater des Bildhauers wird deswegen erwähnt, weil Demosthenes unterstreichen will, daß der Bildhauer auch nicht mit einem Augenzeugen gesprochen haben könnte: Selbst wenn der Großvater bei der Geburt des Bildhauers schon 75 Jahre alt, der Enkel seinerseits bei der Herstellung der Statue genauso alt war $(75+75=150)$, kommt man nicht an die Differenz (190) heran. Aus dem Zusammenhang folgt also ,ganz klar,“ daß sich die Zahl 240 auf das Ereignis bezieht, das den öffentlichen Reden Solons und damit der Gelegenheit, ihm dabei zuzusehen, ein Ende machte, nämlich dessen Ableben. ${ }^{11}$

\footnotetext{
${ }^{7}$ Auf den Fehler machte W. Aly, Solon, RE 3A, 1927, 949 aufmerksam.

${ }^{8}$ Zur Statue Solons vor der Stoa Poikile vgl. M. Sehlmeyer, Stadtrömische Ehrenstatuen der republikanischen Zeit, Stuttgart 1999, 25-26, der im Rahmen seiner Studie einen Überblick über die Verleihung von Ehrenstatuen im spätarchaischen und klassischen Athen (S. 22-27) gibt.

9 „Man darf“ die Herkunftsangabe ,angesichts der bekannten Beziehungen, die S(olon) zu Salamis gehabt hat, bezweifeln“(Aly, Solon, Sp. 948).

${ }^{10}$ Nach R. Thomas, Oral Tradition and Written Record in Classical Athens, Cambridge 1989, 202 A. 20, sei Dem. 19.251 die einzige Passage, in der ein Redner die Geschichtlichkeit einer Behauptung bestreite.

${ }^{11}$ Ein Gutachter fragt, ob von der Akme Solons die Rede sein könne. Mitnichten. Da die antike Überlieferung nicht wußte, wann Solon geboren wurde, so konnte bei ihr für die Akme Solons nur eine einzige Olympiade infrage kommen, nämlich diejenige, in der dessen Gesetzgebung zustande kam. Es sind auch Schriften auf uns gekommen, die Solons Akme erwartungsgemäß in die 46. Olympiade datieren (D.L. 1.62, Clem. Al. Strom.
} 
Die Zahl 240 scheint nun auf den ersten Blick ebenso wenig auf den Heimgang Solons zuzutreffen wie auf die Gesetzgebung: Der Zeitabschnitt ist nicht mehr um 10 Jahre zu kurz, sondern um 20 Jahre zu lang. Denn für Solon liegen ja zwei Todesdaten bei Plutarch (Sol. 32.3) vor: Nach Herakleides Pontikos habe er die Erhebung des Peisistratos eine geraume

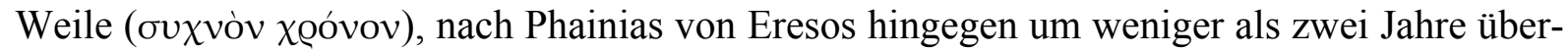
lebt. Letzterer wußte zu berichten, daß Solon im Jahre 560/59 entschlafen sei, was, wie man einräumen muß, ,urkundlich...klingt.“" ${ }^{\text {12 }}$ Die Forschung kennt nur diese beiden Daten, ${ }^{13}$ wir können jedoch nicht aufgrund der Darstellung Plutarchs mit Zuversicht sagen, daß den Athenern im vierten Jahrhundert keine dritte Datierung bekannt gewesen sei. Wir wissen daher zu wenig, um die Zahl 240 als korrupt überliefert hinzustellen, und nicht genug, um in der leichten Rechnung eine Blamage für Demosthenes zu erblicken. Eines ist sicher: Demosthenes tat sein Möglichstes, um seinen Gegner bloßzustellen, und das bedeutet in diesem konkreten Fall, daß er das Intervall von dem Tode Solons bis auf die Errichtung der Statue, so gut es eben ging, ausgedehnt hätte. Darum verbietet es sich, die Statue auf Salamis nach ca. 390 anzusetzen, ${ }^{14}$ und darum kann es nicht wundernehmen, daß Demosthenes die ihm ${ }^{15}$ wie uns bekannten späteren Todesdaten verschwieg und aus den verfügbaren Jahren dasjenige zum Todesjahr erkor, das ihm bei seiner Auseinandersetzung mit Aischines nutzte. Um es einmal klar und deutlich zu sagen: Wir beschäftigen uns gerade mit der einzigen uns erhaltenen Passage, in der das früheste der dem Verfasser derselben bekannten Todesdaten tatsächlich zu erwarten ist. Schauen wir also diesem geschenkten Gaul nicht länger ins Maul!

Es bleibt, den Zeitpunkt des Todes, auf den Demosthenes anspielt, näher zu bestimmen. Die Zahl soll nun nur annähernd richtig sein: ónoṽ, meinte Demosthenes. ${ }^{16}$ Wurde der Tod auch in dieser Version auf ein Archontenjahr gesetzt, könnte man dem Umstand, daß Demosthenes im Jahre 343/2 die Zahl 240 als keine genaue Angabe betrachtete, entnehmen, daß der Tod Solons (bei exklusiver Rechnungsweise) nicht ins Jahr 583/2 gefallen sein könnte. Doch sei dem, wie auch immer - wahrscheinlich ist, daß Demosthenes bei seiner Auseinandersetzung die Zahl 245 aufgerundet hätte. Mithin war die genaue Zahl, die ja wenigstens 235 sein mußte, höchstens 244. Von einer weiteren Präzisierung muß man absehen. Die Quelle läßt nur so viel zu: Solons Tod gehört nach diesem Zeugnis in die Zeit zwischen 587 und 578 v. Chr. Über die Zuverlässigkeit der bisher verkannten Todesnachricht haben wir noch kein Wort verloren. Da aber kein geringerer als Jacoby, der zunächst die Version des Phainias nicht anzweifelte, ${ }^{17}$ sich geraume Zeit später für ein früheres Sterbejahr aussprach, ohne daß er auf

1.65.3, Tatian. Hell. 41; vgl. Suidas s. n.: die 47. od. 56. O1.); hierzu vgl. Jacoby, Chronik 165-67, 173. Aller Wahrscheinlichkeit nach lag nun Solons eigentliche Blüte vor dem Jahr der Gesetzgebung. Doch wie dem auch immer gewesen sein mag — von Bedeutung ist der Umstand, daß Solon noch im Greisenalter als Redner aufgetreten sein könnte.

${ }^{12}$ Aly, Solon, Sp. 949.

${ }^{13}$ Vgl. Jacoby, Chronik 173-74; Aly, Solon, Sp. 949; J. K. Davies, Athenian Propertied Families 600-300 B.C., Oxford 1971, 323-24.

${ }^{14}$ Vgl. Sehlmeyer, Ehrenstatuen 26: „Dabei dürfte ihm daran gelegen gewesen sein, die Statue möglichst jung erscheinen zu lassen, um den Vorredner Aeschines zu diskreditieren.“"

${ }^{15}$ Phainias war bekanntlich Schüler des Aristoteles. Die aristotelische Verfassungsgeschichte (A.P. 14.2, 17.2) stehe in Einklang mit der Angabe des Phainias: Jacoby, Chronik 173 A. 19; die Datierung des Phainias „is...likely...to be the date assumed by A.P.“: P. J. Rhodes, A Commentary on the Aristotelian Athenaion Politeia, Oxford 1981, 224.

${ }^{16}$ MacDowell (A. 1) übersetzte „two hundred and forty years altogether,“ äußerte sich aber nicht zu dem Adverb in seinem Kommentar; die Wörterbücher geben in diesem Punkt Paulsen (A. 1) Recht, der in seinem Kommentar das Adverb mit „ungefähr“" wiedergibt.

17 Jacoby, Chronik 173-74: „Phanias’ angabe beruht offenbar auf einer Atthis, wie schon die archontennamen zeigen; an ihrer historischen richtigkeit brauchen wir nicht zu zweifeln.“ 
eine Quelle zu verweisen vermochte, ${ }^{18}$ wird man im vorliegenden Falle sagen können, daß die Glaubwürdigkeit der Nachricht unterstrichen wurde, bevor dieselbe eintraf. ${ }^{19}$

Dr. Francis X. Ryan

Technische Universität Dresden, Institut für Geschichte

E-mail:fxryan@gmx.de

\footnotetext{
${ }^{18}$ Jacoby, Patrios nomos: State Burial in Athens and the Public Cemetery in the Kerameikos, JHS 64, 1944 [1946], 50 A. 64: "For my part I become more and more doubtful whether the date of Phainias for the death of Solon... is worth more than the divergent statements about his grave, or the place of his death. I submit that it is possible, even probable, that Solon, after having left Athens (at the time of the tyrant Damasias, 582/0 B.C.?), did not return, but died abroad, perhaps a considerable time before 560.... The date of Phainias is conjectural: it belongs to the Solon-Peisistratos legend." Siehe auch dens., Atthis: The Local Chronicles of Ancient Athens, Oxford 1949, 365 A. 70: "The apparently universal confidence in the documentary character and in the correctness of the date of Solon's death...is due to the preconceived opinion that all archons' dates were taken directly from 'the chronicle'....Actually these dates primarily show only that the Atthides...entered an event under the year in question."

${ }^{19}$ Ein Forschungsstipendium der Alexander von Humboldt-Stiftung hat den zügigen Abschluß dieser Untersuchung ermöglicht.
} 